\title{
Abnormal resting-state functional connectivity of hippocampal subfields in patients with major depressive disorder
}

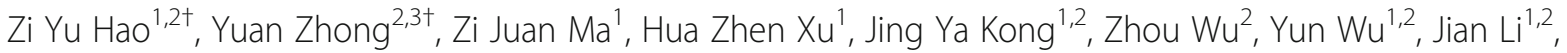
Xin Lu ${ }^{1,2}$, Ning Zhang ${ }^{1,2,4,5}$ and Chun Wang ${ }^{1,2,4,5^{*}}$ (i)

\begin{abstract}
Background: Many studies have found that the hippocampus plays a very important role in major depressive disorder (MDD). The hippocampus can be divided into three subfields: the cornu ammonis (CA), dentate gyrus (DG) and subiculum. Each subfield of the hippocampus has a unique function and are differentially associated with the pathological mechanisms of MDD. However, no research exists to describe the resting state functional connectivity of each hippocampal subfield in MDD.

Methods: Fifty-five patients with MDD and 25 healthy controls (HCs) matched for gender, age and years of education were obtained. A seed-based method that imposed a template on the whole brain was used to assess the resting-state functional connectivity (rsFC) of each hippocampal subfield.

Results: Patients with MDD demonstrated increased connectivity in the left premotor cortex (PMC) and reduced connectivity in the right insula with the CA seed region. Increased connectivity was reported in the left orbitofrontal cortex (OFC) and left ventrolateral prefrontal cortex (VIPFC) with the DG seed region. The subiculum seed region revealed increased connectivity with the left premotor cortex (PMC), the right middle frontal gyrus (MFG), the left ventrolateral prefrontal cortex (VIPFC) and reduced connectivity with the right insula. ROC curves confirmed that the differences between groups were statistically significant.

Conclusion: The results suggest that the CA, DG and subiculum have significant involvement with MDD. Specifically, the abnormal functional connectivity of the CA may be related to bias of coding and integration of information in patients with MDD. The abnormal functional connectivity of the DG may be related to the impairment of working memory in patients with MDD, and the abnormal functional connectivity of the subiculum may be related to cognitive impairment and negative emotions in patients with MDD.
\end{abstract}

Keywords: Major depressive disorder, Hippocampal subfields, Cornu ammonis, Dentate gyrus, Subiculum, Restingstate functional connectivity

\section{Background}

Major depressive disorder (MDD) is a psychiatric illness that seriously affects society and life [34, 43]. MDD affects 350 million people worldwide each year (WHO, 2017), and its lifetime prevalence can reach $3.4 \%$ in

\footnotetext{
* Correspondence: fm51109@163.com

${ }^{\dagger}$ Zi Yu Hao and Yuan Zhong contributed equally to this work.

${ }^{1}$ Nanjing Brain Hospital Affiliated to Nanjing Medical University, Nanjing

210029, Jiangsu, China

${ }^{2}$ School of Psychology, Nanjing Normal University, Nanjing 210097, Jiangsu, China

Full list of author information is available at the end of the article
}

China [76]. The main symptoms of MDD include persistent negative effects, loss of power, inattention and increased guilt and appetite, which are associated with abnormal brain function and structure [5]. Furthermore, the WHO predicts MDD will become the world's second-largest disease by 2020 [46]. However, the pathophysiology of the disease is still unclear and the recurrence rate is very high.

The hippocampus is a core component of the limbiccortical dysregulation model of MDD, which is involved in the foundation of MDD neurobiology and plays a very

(c) The Author(s). 2020 Open Access This article is distributed under the terms of the Creative Commons Attribution 4.0 International License (http://creativecommons.org/licenses/by/4.0/), which permits unrestricted use, distribution, and 
important role in memory and cognitive function [25, $41,51]$. Moreover, the hippocampus also plays an important role in the regulation of stress and emotion [18]. Therefore, the memory deficits and depression experienced by patients with MDD may originate with the hippocampus $[40,58]$. Unsurprisingly, many studies have found abnormal activation of the hippocampus in patients with MDD [32, 44]. These findings indicate that the hippocampus may be involved in the neurobiological basis of MDD.

Recently, it was discovered that the hippocampus can be divided into three different subfields for research. This is because the different subfields of the hippocampus have distinct functions [65]. The three subfields are: the cornu ammonis (CA), dentate gyrus (DG) and subiculum [17] [1]. The CA is related to learning and memory functions in humans and other mammals, and mainly participates in short-term image contact, image formation and fear memory formation. In addition, the CA plays an important role in medium-term and shortterm spatial memory [28]. The DG, on the other hand, is the receptacle for incoming spatial information to the hippocampus. At the same time, it also processes and encodes spatial information, which plays an important role in spatial learning and memory [33]. Meanwhile, the subiculum is the main component of hippocampal information output, transmitting information processed from the DG to the corresponding neuroendocrine system and is also the effector of the baroreceptor reflex [47].

The hippocampus is composed of several subfields that are differentially associated with MDD [72]. However, most studies of MDD in hippocampal subfields focus on volume. Many studies have reported smaller hippocampal subfield volumes compared to healthy controls $[9,10,26]$ however, smaller hippocampal subfield volumes in patients with MDD is not a universal phenomenon, so it is controversial to use hippocampal subfield volume as a biomarker of depression $[3,16,59]$. Therefore, it is not enough to study the changes of hippocampal subfield volume in patients with MDD. We should also explore the differences in functional connectivity of hippocampal subfields between groups.

fMRI research has increased dramatically in recent years, especially in the field of resting-state functional connectivity (rsFC). rsFC represents the temporal coherence of the blood-oxygen-level-dependent (BOLD) signal within or between brain regions or networks during rest, and subsequently helps to reveal the neurobiological basis of MDD [45, 62]. Seeing that many studies have found that some symptoms of MDD are related to abnormal rsFC [30, 69], this article uses the method of resting-state fMRI to explore rsFC of hippocampal subfields.
The aim of this study was to extend our understanding of the role of hippocampal subfields in MDD by examining rsFC of each hippocampal subfield with the whole brain along with differences in rsFC of hippocampal subfields between patients with MDD and HCs. We hypothesized that rsFC of each hippocampal subfield would differ between patients with MDD and HCs and that rsFC would further elucidate the differences between the CA, DG and subiculum.

\section{Method}

\section{Participants}

Patients with MDD were recruited from the Department of Medical Psychology of Nanjing Brain Hospital, affiliated with Nanjing Medical University. HCs were recruited from society through advertising and matched with MDD patients in terms of age, gender and education.

Inclusion criteria for patients with MDD included: (1) conformation to the DSM-IV diagnostic criteria of MDD, (2) 20-50 years old, (3) in their first onset, (4) Scores $\geq 18$ on the 24-item version of the Hamilton Rating Scale for Depression (HAMD, Hamilton M, 1960), (5) right-handed, (6) voluntary participation and signed informed consent. Exclusion criteria for patients included: (1) patients with other psychotic disorders, severe physical illness or infectious diseases, (2) substance abuse, (3) current pregnancy, (4) MRI contraindications, (5) patients who received systemic drug therapy, psychotherapy or electroconvulsive therapy within 6 months prior to enrollment.

HCs met the following criteria: (1) 20-50 years old, (2) right handed, (3) voluntary participation, and signed informed consent. The exclusion criteria for HCs included: (1) people with nervous system disease, mental illness or serious physical illness, (2) personal history/family history of psychiatric disorders or psychiatric illness, (3) have taken psychotropic drugs or had psychological counseling within the past 3 months, (4) current pregnancy, (5) MRI contraindications.

\section{fMRI data acquisition and processing}

All rs-fMRI data were acquired on a Siemens Verio MRI 3.0 Tesla scanner. Before scanning, foam pads and earplugs were used to reduce head movement and noise. All subjects were instructed to stay awake and close their eyes during the scan. In order to reduce data errors, subjects whose heads exceeded $3^{\circ}$ of motion were rejected. fMRI scanner parameters: gradient-echo and echo-planar-imaging, T1-weighted structure image, TR = $1900 \mathrm{~ms}, \mathrm{TE}=2.48 \mathrm{~ms}$, flip angle $=90^{\circ}, \mathrm{FOV}=250 \mathrm{~mm}$, matrix $=256 \times 256,176$ slices, slices thickness $/$ gap $=1.0$ $\mathrm{mm} / 0.5 \mathrm{~mm}$. T2-weighted functional image: $\mathrm{TR}=3000$ $\mathrm{ms}, \mathrm{TE}=40 \mathrm{~ms}$, flip angle $=90^{\circ}, \mathrm{FOV}=240 \mathrm{~mm}$, matrix $=$ 
$64 \times 64,32$ slices, slice thickness $/$ gap $=4 \mathrm{~mm} / 4 \mathrm{~mm}$, total time $=8 \min 6 \mathrm{~s}$.

Rs-fMRI data were preprocessed using Data Processing Assistant of Resting State fMRI (DPARSF) within the MATLAB toolbox, which is based on SPM (Statistical Parametric Mapping) and REST (Resting-State fMRI Data Analysis Toolkit) (http://restfmri.net/forum/). Data preprocessing included: realignment and head motion correction (head motion or rotation greater than $3^{\circ}$ would be excluded), spatial normalization (the functional images were spatially normalized to MNI (Montreal Neurological Institute) template and resampled to $3 * 3 * 3$ $\mathrm{mm} 3$ ) and smoothing (full width at half maximum, FWHM $\left.=6 \mathrm{~mm}^{*} 6 \mathrm{~mm}^{*} 6 \mathrm{~mm}\right)$. Then, detrending and filtering $(0.01 \sim 0.08)$ were used to remove high-frequency physiological noise and low-frequency drift. We also regressed out nuisance covariates including 6 head motion parameters, global mean signal, cerebrospinal fluid signal and white matter signal. The group differences of head motion were assessed with the two sample t-test according to the following formula:

$$
\text { Head Motion/Rotation }==\quad \frac{1}{L-1} \sum_{i=2}^{L}
$$
$\sqrt{\left|x_{i}-x_{i-1}\right|^{2}+\left|y_{i}-y_{i-1}\right|^{2}+\left|z_{i}-z_{i-1}\right|^{2}}$ [78]. The results indicated that there was no significant distinction between the two groups (two sample t-test, $t=-1.3101, p=$ 0.1943 for translation, and $t=-1.3132, p=0.1933$ for rotational).

\section{Hippocampal subfields definition}

The hippocampal subfield template we used divided the hippocampus into three subfields: CA, DG and subiculum (Fig. 1). The hippocampal subfields were divided by probabilistic maps that had organizational structure boundaries. Then, they were resliced according to the spatial voxel size of $3 \mathrm{~mm} \times 3 \mathrm{~mm} \times 3 \mathrm{~mm}$ and selected so that at least $50 \%$ of the voxels fell into the seed area to make the final seed area. Each voxel belonged to only one seed area. Then the time course of the seed area was extracted after averaging the BOLD signals of the bilateral seed areas, respectively, for the following functional connectivity analysis.

\section{Data analysis}

There were no significant differences in age, years of education or gender between groups at a significance level of $P<0.05$. In contrast, there were significant differences in HAMD score between the two groups (Table 1). Analyses were based on the Statistical Package for the Social Sciences25 (SPSS25) (https://www.ibm. com/analytics/spss-statistics-software).

After processing, individual- subject level voxel-wise analyses were built in the REST toolbox (Song et al., 2011) by using Fisher's r-to- $Z$ transform to convert the data to Z-scores. In order to identify the differences in rsFC of each hippocampal subfield with the whole brain and the differences in rsFC of hippocampal subfields between patients with MDD and $\mathrm{HCs}$, rsFC analysis was performed using the second-level model in SPM8.

Finally, the ROC curve, which can discriminate patients with major depressive disorder from healthy controls, was drawn by using $\mathrm{Z}$ scores of hippocampal subfield rsFC with between-group differences. The ROC curve was done in SPSS25, with a larger area under the curve (AUC) providing more accurate results. The $\mathrm{Z}$ scores of rsFC that were extracted from each voxel in significant clusters were also used to test the correlation between HAMD scores and functional connections through Pearson linear partial correlation at 95\% confidence level in SPSS25.

\section{Results}

\section{Demographics}

Demographic and clinical data were collected from participants upon recruitment. Patients with MDD and HCs

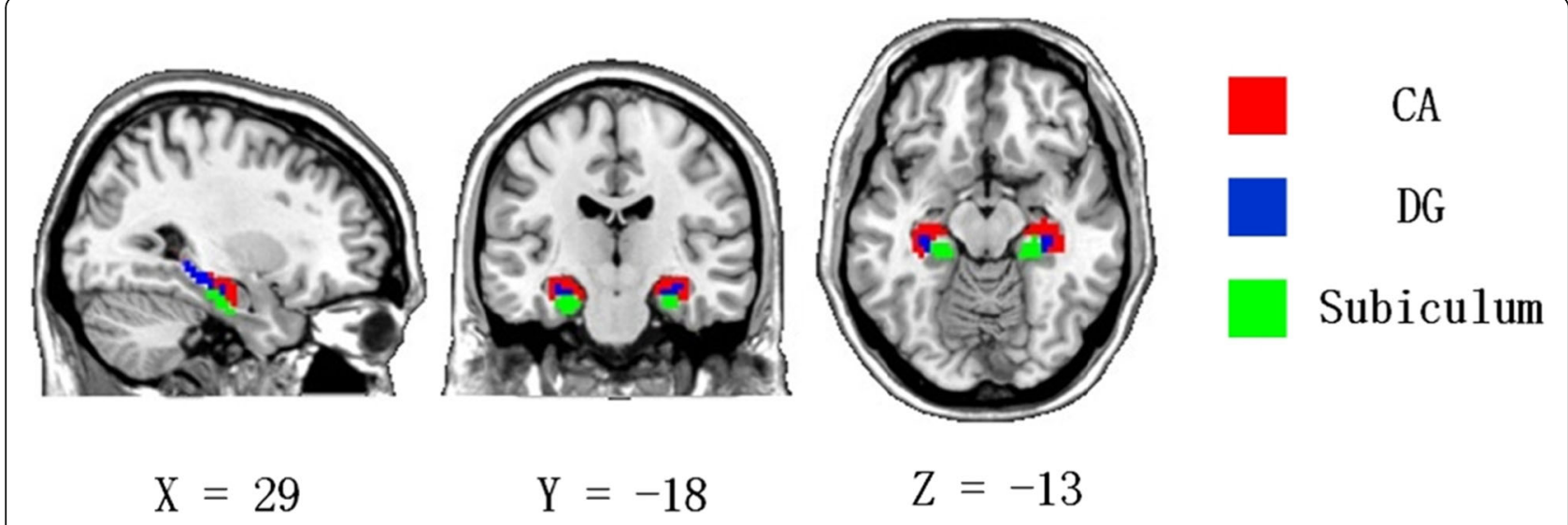

Fig. 1 Hippocampal subfields. CA, cornu ammonis (shown in red); DG, dentate gyrus (shown in blue); subiculum (shown in green) 
Table 1 Comparison of demographic and clinical variables among MDD and HCs

\begin{tabular}{lllll}
\hline Variable & $\mathrm{MDD}(n=55)$ & $\mathrm{HC}(n=25)$ & $\mathrm{t} / \mathrm{x} 2$ & $\mathrm{p}$ \\
\hline Gender (males/females) & $20 / 35$ & $13 / 12$ & 1.315 & 0.193 \\
Age & $34.80 \pm 9.04$ & $38.24 \pm 10.13$ & -1.561 & 0.123 \\
Education & $14.12 \pm 2.03$ & $14.32 \pm 2.75$ & -0.329 & 0.744 \\
HAMD & $25.00 \pm 5.16$ & $2.72 \pm 1.46$ & 29.548 & 0.000 \\
\hline
\end{tabular}

MDD major depressive disorder, $H C s$ healthy controls, HAMD Hamilton Depression Scale

were compared in terms of gender, age, HAMD scores and years of education. While there were no significant differences in gender, age or years of education, there was a significant difference in HAMD scores; HAMD scores in patients with MDD were significantly higher than HCs (Table 1).

\section{RsFC of hippocampal subfields}

Based on results from the one-sample t test, we observed positive functional connectivity between the hippocampal subfields (CA, DG and subiculum) and a wide range of brain regions including the hippocampus, lingual gyrus, inferior temporal gyrus, amygdala, middle occipital gyrus, orbitofrontal cortex (OFC) and medial prefrontal cortex (mPFC). Negative functional connectivity was noted between the hippocampal subfields and the insula, posterior parietal cortex (PPC) and dorsolateral prefrontal cortex (dlPFC) (Fig. 2 and Table 2).

Results from the one-sample $t$ test also revealed that there were differences in the rsFC maps of the CA, DG and subiculum. For instance, positive functional connectivity was exhibited by the anterior cingulate cortex (ACC) with the CA seed region. In addition, negative functional connectivity was reported between the DG seed and the left cerebellum. Finally, negative functional connectivity was demonstrated between the subiculum seed and the medial frontal cortex (MFC) and right premotor cortex (PMC) (Fig. 2). The results were corrected using the FDR method (threshold of $P<0.01$ ).

\section{RsFC alterations between patients with MDD and HCs}

Compared to $\mathrm{HCs}$, the $\mathrm{CA}$ seed region revealed increased functional connectivity with the left PMC in MDD. On the other hand, reduced functional connectivity between the CA and the right insula was also shown. Furthermore, increased functional connectivity was reported between the DG seed and the left OFC and left vIPFC. With the subiculum seed region, increased functional connectivity was revealed between the left PMC, the right MFG and the left vlPFC. In addition, decreased functional connectivity was shown between the subiculum and the right insula (Fig. 3 and Table 3). Results were corrected using the AlphaSim method (threshold of $P<0.001$, cluster: $p<0.05)$.
ROC curves can effectively distinguish between patients with MDD and HCs by measuring area under the curve (AUC), with larger areas providing greater accuracy. In this study, the AUC of the ROC curves of the left $\mathrm{PMC}$ and the right insula were 0.76 and 0.75 , respectively, when the CA was the seed region. The AUC of the ROC curves of the left OFC and the left vlPFC were 0.82 and 0.76 , repectively, when the DG was the seed region. The AUC of the ROC curves of the left PMC, the MFG, the left vlPFC and the right insula were 0.79, 0.77, 0.77 and 0.76 , repectively, when the subiculum was the seed region. In addition, the cutoff point for rsFC altertions in hippocampal subfields was more than 1.32 and the cutoff point for the left OFC with the DG seed region was as high as 1.63 , which means that the functional circuits of hippocampal subfields have potential to aid in clinical diagnosis (Fig. 4).

\section{Discussion}

This study was designed to assess differences between patients with MDD and HCs in functional circuitry of each hippocampal subfield, the CA, DG and subiculum, based on whole-brain rsFC. This article demonstrated that patients with MDD and healthy controls differed in the rsFC of each hippocampal subfield. Specifically, patients with MDD primarily displayed alterations between hippocampal subregions and the PMC, OFC, vlPFC, MFG and insula.

In MDD patients and HCs, our findings of positive functional connectivity between hippocampal subfields and the hippocampus, lingual gyrus, inferior temporal gyrus, amygdala, middle occipital gyrus, OFC and mPFC are consistent with previous studies $[6,13,35,52,53$, $74]$. Both the hippocampus and the amygdala are part of the limbic system, thus they are involved in emotional and social processes, along with the temporal gyrus [19]. Although, compared to those brain regions, the $\mathrm{mPFC}$ has a stronger ability to regulate emotions and cognition as part of the default mode network (DMN) [66]. Moreover, the temporal gyrus can be combined with the occipital gyrus, OFC and insula to act together on mood regulation and emotional processes $[13,19]$. Finally, the lingual gyrus is mainly related to verbal memory and facial emotion recognition [36]. Also consistent with previous studies are our findings of negative functional connectivity between the three hippocampal subfields (CA, DG and subiculum) and the insula, PPC and dIPFC (X. H $[11,45]$.$) . The PCC belongs to the posterior$ DMN, which mainly participates in the process of consciousness and memory through its relationship with the hippocampus $[2,38]$. The vlPFC is part of the central executive network (CEN), which plays a vital role in cognitive tasks with a variety of attentional requirements [39]. All of these findings demonstrate that the CA, DG, and 
$\mathrm{HC}$
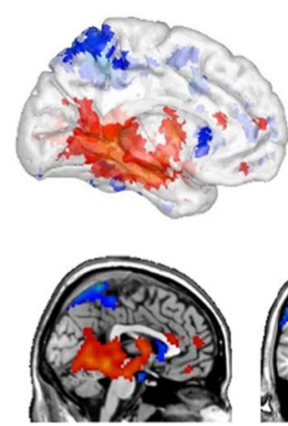

$\mathrm{X}=-1$

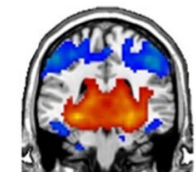

$\mathrm{Y}=-36$

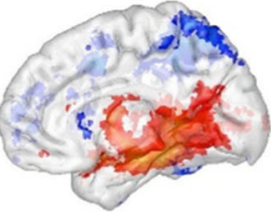

$\mathrm{CA}$

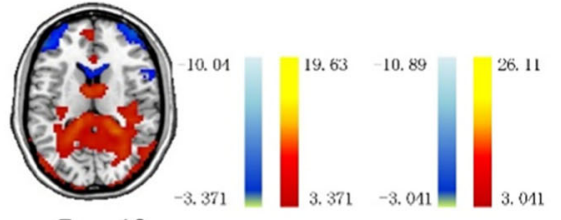

$Z=13$

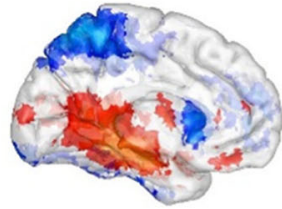

MDD

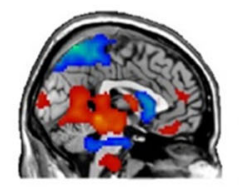

$\mathrm{X}=-1$

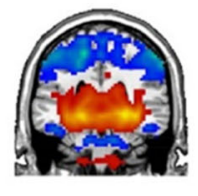

$\mathrm{Y}=-36$

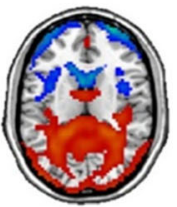

$Z=13$
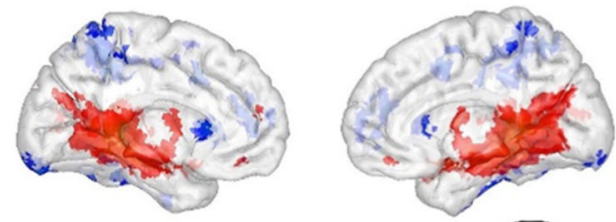

DG
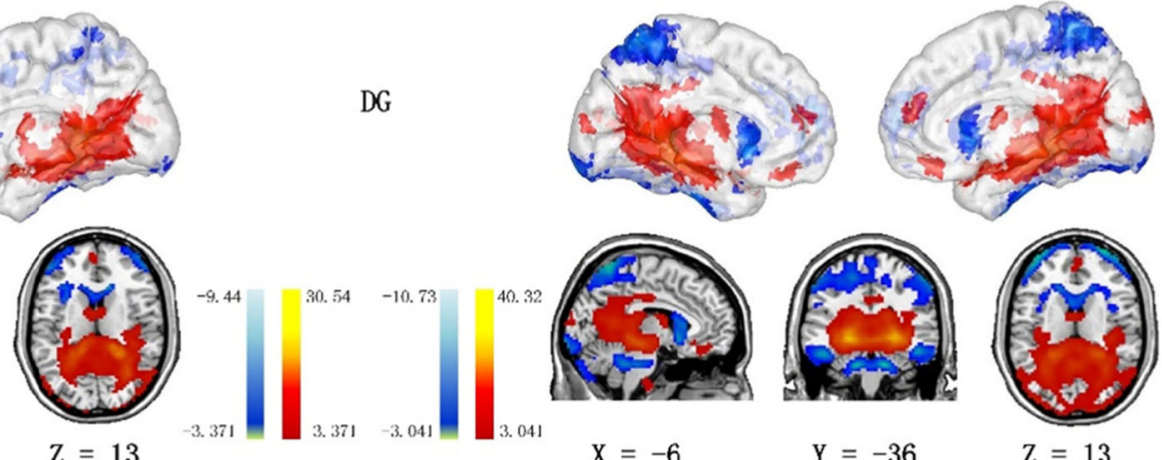

$X=-6$

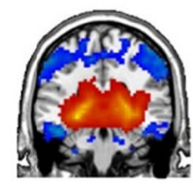

$\mathrm{Y}=-36$

$Z=13$
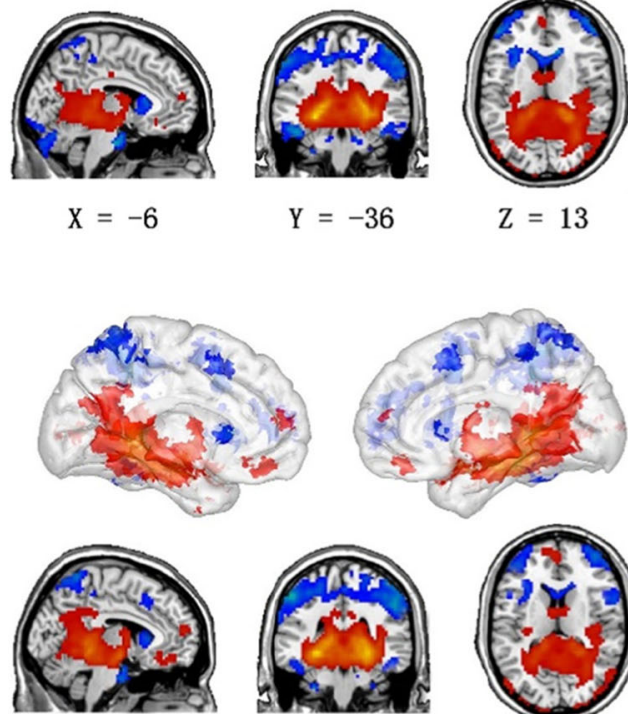

$\mathrm{Y}=-36$

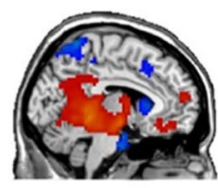

$X=-6$

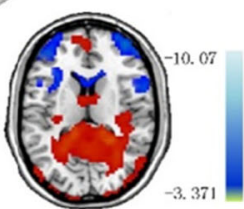

$Z=13$

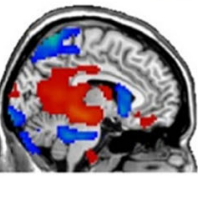

$\mathrm{X}=-6$

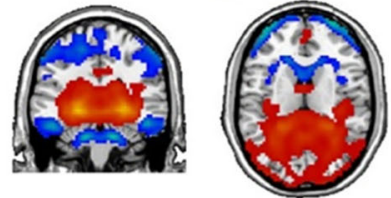

$\mathrm{Y}=-36$

$\mathrm{Z}=13$

Fig. 2 Functional connectivity maps of each hippocampal subfield in MDD and HCs. The results were corrected using the FDR method (threshold of $\mathrm{P}<0.01$ ). Color-bar represents t-values

subiculum are involved in emotion and cognitive regulation, suggesting that disruption of those functional circuits affects cognition, memory and emotion.

Importantly, there were some differences between the rsFC maps of the CA, DG and subiculum. For instance, the CA demonstrated positive functional connectivity with the ACC. The ACC plays a very important role in working memory [57], which is similar to the function of the CA. The DG displayed negative functional connectivity with the left cerebellum, which has recently been discovered to be associated with visual memory and has the ability to encode visuospatial information [7]. Finally, negative functional connectivity was shown between the subiculum and the MFC and right PMC. The MFC is a region involved in action and motor generation [14]. Similarly, the PMC also has the ability to generate motor plans. Therefore, these differences between the rsFC maps of the CA, DG and subiculum must be caused by the unique function of each hippocampal subfield.

Selecting the CA and subiculum as seed regions revealed increased functional connectivity of the left PMC in patients with MDD compared to HCs. The PMC is 
Table 2 Resting-state functional connectivity of each hippocampal subfield

\begin{tabular}{|c|c|c|c|c|}
\hline Seed & Brain region & $\mathrm{BA}$ & voxel & $\mathrm{t}$ \\
\hline \multirow[t]{10}{*}{$\overline{C A}$} & $\mathrm{mPFC}$ & 10 & 35 & 5.51 \\
\hline & Lingual Gyrus & 29 & 256 & 19.63 \\
\hline & Temporal_Inf_L & 21 & 115 & 19.63 \\
\hline & Amygdala & 34 & 75 & 19.63 \\
\hline & Hippocampus & 35 & 75 & 19.63 \\
\hline & Occipital_Mid_R & 19 & 122 & 19.63 \\
\hline & ACC & 24 & 34 & 5.80 \\
\hline & dIPFC & 10 & 306 & -6.09 \\
\hline & Insula & 13 & 37 & -9.33 \\
\hline & PPC & 40 & 233 & -9.33 \\
\hline \multirow[t]{11}{*}{ DG } & mPFC & 11 & 37 & 4.62 \\
\hline & Lingual Gyrus & 29 & 328 & 30.53 \\
\hline & Temporal_Mid_R & 21 & 280 & 30.53 \\
\hline & Middle Occipital Gyrus & 19 & 172 & 30.53 \\
\hline & Amygdala & 34 & 76 & 30.53 \\
\hline & Hippocampus & 35 & 75 & 30.53 \\
\hline & Left Cerebellum & & 220 & -7.92 \\
\hline & dIPFC & 10 & 148 & -7.51 \\
\hline & Insula & 13 & 30 & -5.85 \\
\hline & MFG & 40 & 474 & -8.35 \\
\hline & PPC & 40 & 281 & -8.35 \\
\hline \multirow[t]{12}{*}{ Subiculum } & OFC & 11 & 21 & 6.04 \\
\hline & $\mathrm{mPFC}$ & 10 & 50 & 5.45 \\
\hline & Middle Occipital Gyrus & 19 & 211 & 21.20 \\
\hline & Lingual Gyrus & 29 & 204 & 21.20 \\
\hline & Temporal_Inf_L & 21 & 111 & 21.20 \\
\hline & Amygdala & 34 & 77 & 21.20 \\
\hline & Hippocampus & 35 & 75 & 21.20 \\
\hline & dIPFC & 10 & 181 & -8.57 \\
\hline & MFC & 9 & 85 & -8.57 \\
\hline & PMC & 6 & 73 & -8.57 \\
\hline & Insula & 13 & 62 & -8.57 \\
\hline & PPC & 40 & 334 & -10.07 \\
\hline
\end{tabular}

mPFC medial prefrontal cortex, $A C C$ anterior cingulate cortex, dIPFC dorsolateral prefrontal cortex, $P P C$ posterior parietal cortex, MFG medial frontal gyrus, OFC orbitofrontal cortex, MFC medial frontal cortex, PMC premotor cortex

generally understood to convert visuospatial information into a motor plan specific to the position and shape of the object $[22,31,42]$. As mentioned above, the CA is mainly involved in the formation of image and spatial memory, similar to the role of the PMC [28]. This helps to explain the increased functional connectivity we observed between the CA and PMC. Moreover, the PMC can also generate avoidance motivation according to expected danger [15], which may be related to the avoidance behavior displayed by patients with MDD. The CA and subiculum seed regions also revealed decreased rsFC in the right insula. The insula is a core brain area that is primarily responsible for integrating cognitive and emotional information [60]. In addition, the dysfunction of insular has been found in psychosis spectrum disorders, especially in the first-episode depression patients. Moreover, the insular cortex is related to the cognitiveaffective function [64]. Therefore, the dysfunction of insular cortex will destroy the cognitive and emotional function of depression patients. The insula is also part of the hate circuit, which exhibits reduced activation in response to both positive and negative emotional stimuli, which is also consistent with the results of our study [63]. In addition, the insula is considered a biomarker of MDD, and many studies have found that the functional and structural abnormalities presented by the insula are related to MDD [23, 77]. Therefore, the abnormal connectivity between the $\mathrm{CA}$ and the subiculum with the PMC and insula may be related to the symptoms of MDD, providing evidence for the pathogenesis of MDD. Both of these findings are consistent with previous research [4, 21, 45, 49, 68, 70, 75].

The DG seed region revealed increased functional connectivity with the left OFC. The similar functions of the hippocampus and OFC may explain this [71]; for example, since both the hippocampus and the OFC can predict what is going to happen based on the current situation $[8,55]$, the abnormal connectivity between the DG and OFC may prompt MDD patients to worry excessively about the future. Also, because the OFC is on the receiving end of hippocampal input while the DG is the on receiving end of spatial information into the hippocampus, this relationship further serves to explain why functional connectivity between the DG and OFC is heightened [29, 33]. In addition, many anatomical studies have found that OFC has abnormal gray matter volume. Compared with healthy people, OFC volume of depression patients is smaller. The study also found that Subjects with OFC dysfunction showed personality changes, including behavioral inhibition, emotional instability and reduced motivation [79]. Therefore, it can be concluded that the abnormal connection between DG and OFC may be the main cause of depression patients' low motivation.

When the DG and subiculum were used as seed regions, increased functional connectivity was shown in the left vlPFC. It comes as no surprise then, that many studies have found that the vlPFC plays an important role in working memory and other cognitive functions $[50,54,67]$. In particular, the left VLPFC is involved in the assessment of emotion and conscious impulse control. So, impaired function of vlPFC can lead to 

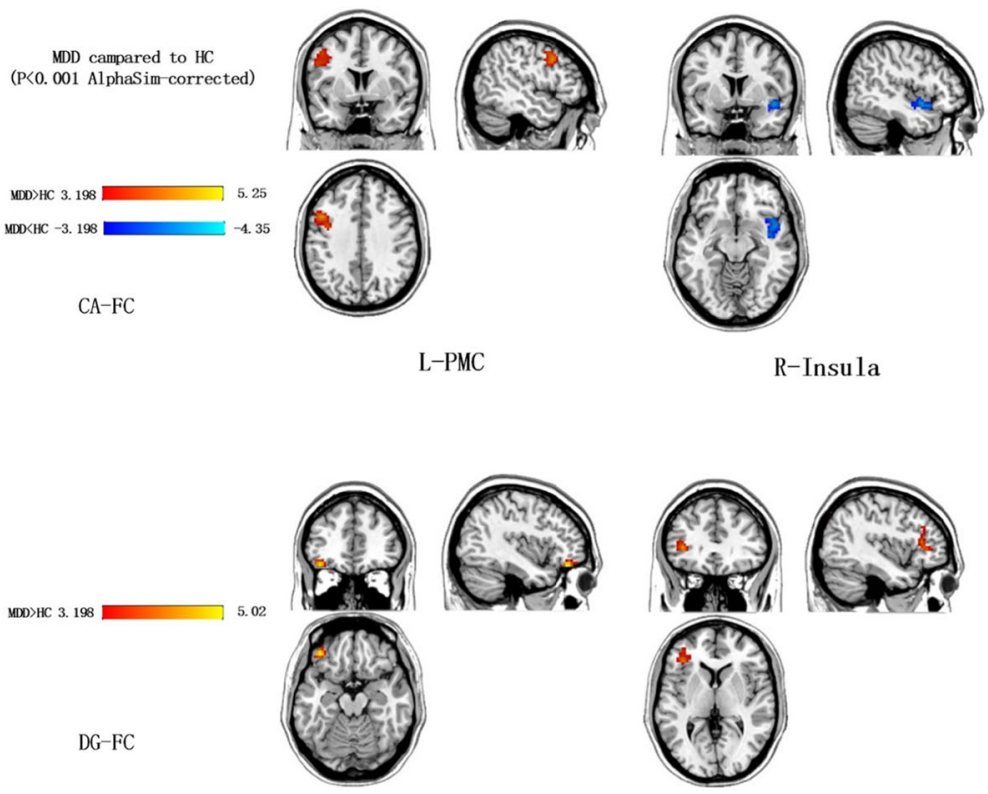

L-OFC

L-v1PFC
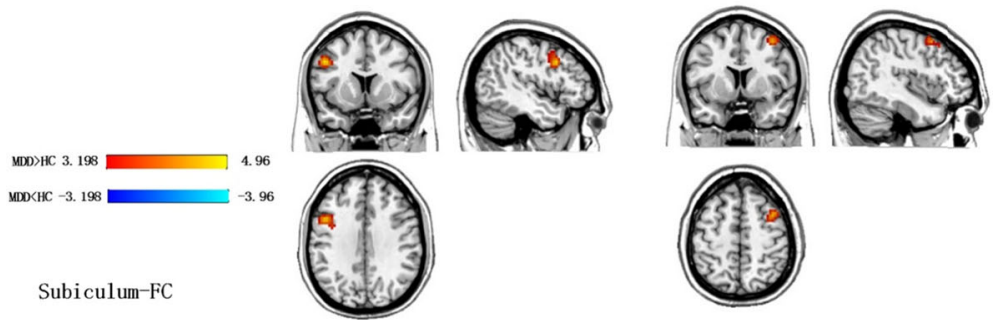

L-PMC

$R-M F G$

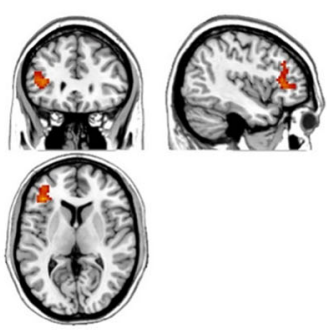

L-vlPFC

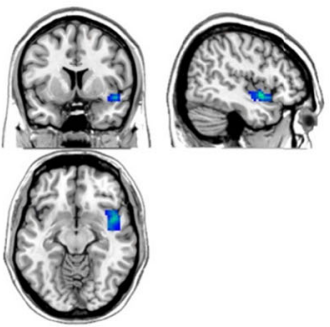

R-Insula

Fig. 3 The rsFC alterations of each hippocampal subfield between MDD and HCs, as determined using a two-sample t-test. Warm colors show increased resting-state functional connectivity and cool colors show decreased resting-state functional connectivity with each hippocampal subfield compared to HC. The color bar represents t-values. MDD: major depressive disorder; HC: healthy control; L-PMC: the left premotor cortex; R-insula: the right insula; L-OFC: the left orbitofrontal cortex; L-vIPFC: the left ventrolateral prefrontal cortex; R-MFG: the right middle frontal gyrus

abnormal emotional assessment and excessive self inhibition in patients with depression [20]. And, the vlPFC is responsible for producing a negative emotional experience [37], so excessive activation of the vlPFC can produce unpleasant emotions. Therefore, this abnormal connectivity may give rise to memory loss and negative emotions in patients with MDD.

Finally, with the subiculum seed region, increased functional connectivity with the right MFG was found and this finding is consistent with previous research [56]. The right MFG plays a very important role in attention as the point of convergence between the dorsolateral prefrontal cortex and the ventrolateral prefrontal cortex [27]. In addition, the MFG is also involved in regulating emotion/cognition and contingency awareness $[12,48]$. At the same time, the research shows that MFG, as a part of DLPFC, also has abnormal gray matter volume, and the dysfunction of this cortex may result in 
Table 3 Resting-state functional connectivity of each hippocampal subfield between MDD and HCs

\begin{tabular}{llllll}
\hline Seed & Brain region & BA & Cluster size & t & $\begin{array}{c}\text { Peak MNI coordinates } \\
X, Y, Z\end{array}$ \\
\hline CA & & & 23 & 3,87 & $45,6,-9$ \\
& L-PMC & 9 & 20 & -3.63 & $-45,12,36$ \\
DG & R-Insula & 38 & 18 & 5.02 & $-39,-39,18$ \\
& L-OFC & 11 & 15 & 3.80 & $-36,33,3$ \\
Subiculum & L-VIPFC & & 4.64 & $-48,12,36$ \\
& L-PMC & 9 & 17 & 4.07 & $39,9,54$ \\
& R-MFG & 6 & 14 & 4.08 & $-39,33,6$ \\
& L-VIPFC & & -3.84 & $45,3,-9$ \\
\hline
\end{tabular}

MDD major depressive disorder, HCS healthy controls, L-PMC the left premotor cortex, $R$-insula the right insula, $L$-OFC the left orbitofrontal cortex, $L$-vIPFC the left ventrolateral prefrontal cortex, $R-M F G$ the right middle frontal gyrus

\section{ROC}

$\mathrm{CA}$
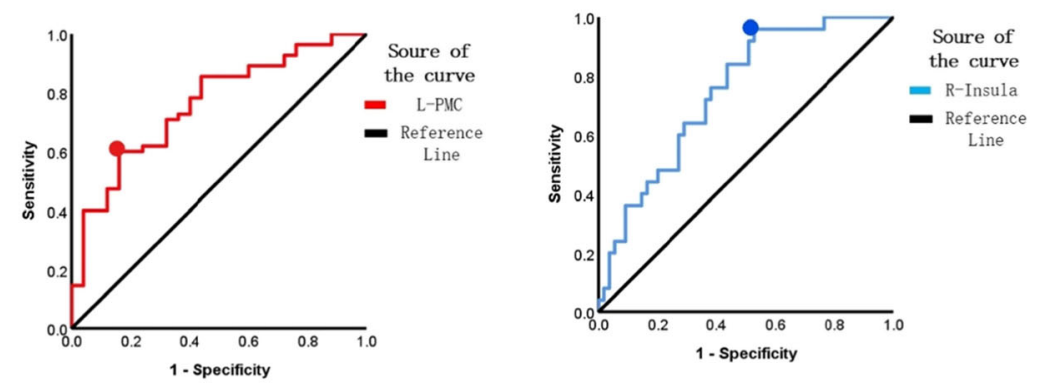

DG

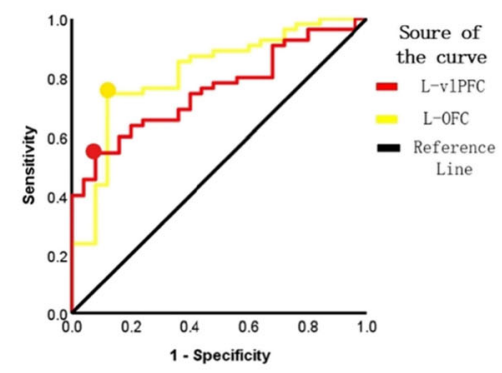

Subiculum
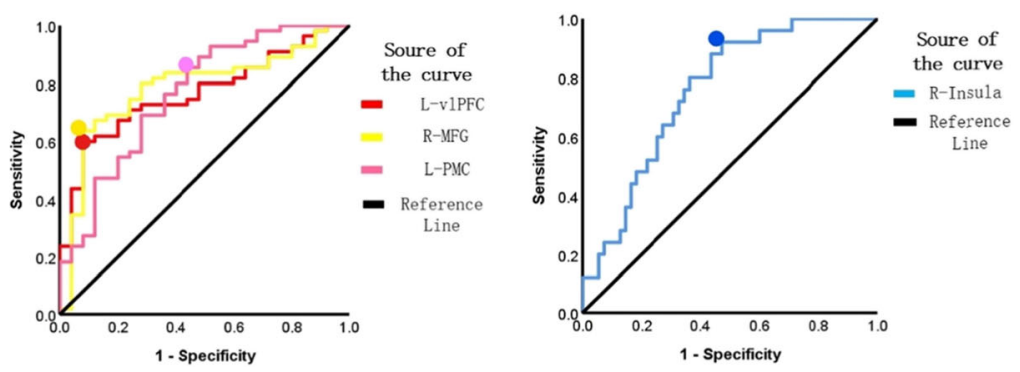

Fig. 4 Receiver operating characteristic (ROC) curve discriminated MDD patients from HCs by evaluating Z scores of rsFC with significant between-group differences; enhanced accuracy was noted with a larger area under the curve (AUC). With the CA seed region, L-PMC: the left premotor cortex (AUC:0.76; cutoff: sensitivity:0.60; specificity:0.84); R-insula: the right insula (AUC:0.75; cutoff: sensitivity:0.96; specificity:0.48). With the DG seed region, L-VIPFC: the left ventrolateral prefrontal cortex (AUC:0.76; cutoff: sensitivity:0.55; specificity:0.92); L-OFC: the left orbitofrontal cortex (AUC:0.82; Cutoff: sensitivity:0.75; specificity:0.88). With the subiculum seed region, L-vIPFC: the left ventrolateral prefrontal cortex (AUC:0.77; cutoff: sensitivity:0.40; specificity:0.92); R-MFG: the right middle gyrus (AUC:0.77; cutoff: sensitivity:0.64; specificity:0.92); L-PMC: the left premotor

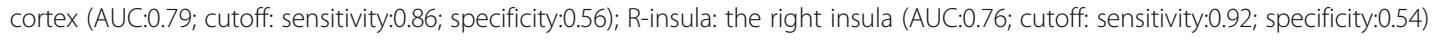


damage in retrieval of long-distance memory, management of external stimulation, appropriate change of behavior and psychological flexibility [79]. Therefore, the abnormal connectivity between the subiculum and the right MFG may be related to the lack of concentration, cognitive dysfunction and excessive worry and tension experienced by patients with MDD. What's more, the subiculum and MFG have similar functions, further explaining the increase in functional connectivity.

All of the above findings prove that the CA, DG and subiculum have unique functions and connections with MDD. Moreover, the abnormal connectivity between each hippocampal subfield with other brain regions causes a series of symptoms such as avoidance behavior in life, low self-evaluation, excessive worry about the future, memory loss, tension and bad mood in patients with MDD. More importantly, through the ROC curve, we are more convinced that each hippocampal subfield plays a very important role in the neurobiological basis of MDD. Therefore, the abnormal connectivity between hippocampal subfields and other brain regions may be used as a biomarker for MDD.

This article has some limitations. First, the CA can be divided into CA1-CA4, but since the $3 \mathrm{~T}$ MRI scanners we use are lower in resolution, we cannot accurately distinguish the difference in functional connectivity between CA1-CA4. It is hoped that the $7 \mathrm{~T}$ MRI can be used in future studies to distinguish the differences between CA1-CA4. Second, the samples we used included only Chinese subjects, so we anticipate that future research can be combined with data from the brainnetome program. Third, this study did not explore the gender differences of functional connections in the hippocampus, so we should pay more attention to gender differences in the future research. Finally, our findings did not correlate with HAMD. It is possible that because most health controls entered the closed environment of the MRI for the first time, we did provoke their frightened and restless instead of MDD patients, thus resulting is an insignificant relationship between the HAMA score with the rsFC of abnormal brain areas in patients with MDD. Therefore, in future research, we should pay more attention to providing emotional guidance to the participants, so that all participants can maintain a peaceful mood, which will make the research results more accurate.

\section{Conclusion}

In conclusion, this study was designed to investigate rsFC of each hippocampal subfield: the CA, DG and subiculum, with the whole brain as well as their rsFC alterations between patients with MDD and HCs. The findings demonstrate that there were rsFC differences between the CA, DG and subiculum and they each have significant relationships with MDD. Thus, this paper emphasizes the importance and contribution of each hippocampal subfield to MDD, as they may be involved in its pathogenesis and can be used as a biomarker to support the clinical diagnosis, treatment and future research of MDD.

\section{Abbreviations}

ACC: anterior cingulate cortex; AUC: Areas under curves; CA: Cornu ammonis; DG: Dentate gyrus; dIPFC: Dorsolateral prefrontal cortex; DSM-IV: Diagnostic and statistical manual of mental disorders; EPI: Echo planar imaging; FA: Flip angle; FOV: Field of view; FWHM: Full width at half maximum; HAMA: Hamilton anxiety rating scale; HCs: Health controls; MDD: Major depressive disorder; MFC: Medial frontal cortex; MFG: Medial frontal gyrus; MNI: Montreal Neurological Institute; mPFC: Medial prefrontal cortex; OFC: Orbitofrontal cortex; PMC: Premotor cortex; PPC: Posterior parietal cortex; ROC: Receiver operating characteristic; SPSS25: Statistical package for the social sciences25; TE: Echo time; TR: Repetition time; vIPFC: Ventrolateral prefrontal cortex

\section{Acknowledgments}

The authors would like to thank Nanjing Brain Hospital, affiliated with Nanjing Medical University of China for their funding and support.

\section{Authors' contributions}

CW designed the study along with NZ. ZH, ZM, JY K, ZW, YW, JL and XL collected and provided patients. ZH managed and analyzed the imaging date. $\mathrm{YZ}$ gave the guidance in date analysis. $\mathrm{ZH}$ wrote the first draft of manuscript. CW revised the draft. All authors contributed to and had approved the final manuscript.

\section{Funding}

This study was supported by National Natural Science Foundation of China (81571344, 81871344); Natural Science Foundation of Jiangsu Province (BK20161109); the Natural Science Foundation of the Higher Education Institutions of Jiangsu Province, China (18KJB190003); key research and development program (Social Development) project of Jiangsu province (BE20156092015); "Six Talent Peak" High-Level Talent Selection and Training Plan of Jiangsu Province (2018-WSN-109).

\section{Availability of data and materials}

The datasets resting state fMRI scans used and analyzed during the current study are available from the corresponding author on reasonable request.

\section{Ethics approval and consent to participate}

All procedures performed in studies involving human participants were in accordance with the ethical standards of the institutional and/or national research committee and with the 1964 Helsinki declaration and its later amendments or comparable ethical standards.

And this research was approved by the Medical Research Ethics Committee of Nanjing Brain Hospital affiliated to Nanjing Medical University. Written informed consent was obtained from all individual participants included in the study.

\section{Consent for publication}

Not applicable.

Competing interests

The authors declare that they have no competing interests.

\section{Author details}

${ }^{1}$ Nanjing Brain Hospital Affiliated to Nanjing Medical University, Nanjing 210029, Jiangsu, China. ${ }^{2}$ School of Psychology, Nanjing Normal University, Nanjing 210097, Jiangsu, China. ${ }^{3}$ Jiangsu Key Laboratory of Mental Health and Cognitive Science, Nanjing Normal University, Nanjing 210097, People's Republic of China. ${ }^{4}$ Functional Brain Imaging Institute of Nanjing Medical University, Nanjing 210029, Jiangsu, China. ${ }^{5}$ Cognitive Behavioral Therapy Institute of Nanjing Medical University, Nanjing 210029, Jiangsu, China. 


\section{Received: 22 August 2019 Accepted: 10 February 2020}

\section{Published online: 17 February 2020}

\section{References}

1. Amunts K, Kedo O, Kindler M, Pieperhoff P, Mohlberg H, Shah NJ, et al. Cytoarchitectonic mapping of the human amygdala, hippocampal region and entorhinal cortex: intersubject variability and probability maps. Anat Embryol (Berl). 2005;210(5-6):343-52. https://doi.org/10.1007/s00429-0050025-5.

2. Andrews-Hanna JR, Smallwood J, Spreng RN. The default network and selfgenerated thought: component processes, dynamic control, and clinical relevance. Year Cognitive Neurosci. 2014;1316:29-52. https://doi.org/10. $1111 /$ nyas. 12360

3. Arnone D, McIntosh AM, Ebmeier KP, Munafo MR, Anderson IM. Magnetic resonance imaging studies in unipolar depression: systematic review and meta-regression analyses. Eur Neuropsychopharmacol. 2012;22(1):1-16. https://doi.org/10.1016/j.euroneuro.2011.05.003.

4. Avery JA, Drevets WC, Moseman SE, Bodurka J, Barcalow JC, Simmons WK Major depressive disorder is associated with abnormal interoceptive activity and functional connectivity in the insula. Biol Psychiatry. 2014;76(3):258-66. https://doi.org/10.1016/j.biopsych.2013.11.027.

5. Belmaker RH, Agam G. Major depressive disorder. N Engl J Med. 2008;358: 55-68.

6. Blum S, Habeck C, Steffener J, Razlighi Q, Stern Y. Functional connectivity of the posterior hippocampus is more dominant as we age. Cogn Neurosci. 2014:5(3-4):150-9. https://doi.org/10.1080/17588928.2014.975680.

7. Brissenden JA, Tobyne SM, Osher DE, Levin EJ, Halko MA, Somers DC. Topographic Cortico-cerebellar networks revealed by visual attention and working memory. Curr Biol. 2018;28(21):3364. https://doi.org/10.1016/j.cub. 2018.08.059.

8. Buckner RL. The role of the Hippocampus in prediction and imagination Annu Rev Psychol. 2010;61:27-48. https://doi.org/10.1146/annurev.psych.60. 110707.163508.

9. Cao B, Luo Q, Fu Y, Du L, Qiu T, Yang X, et al. Predicting individual responses to the electroconvulsive therapy with hippocampal subfield volumes in major depression disorder. Sci Rep. 2018:8(1):5434. https://doi. org/10.1038/s41598-018-23685-9.

10. Cao B, Passos IC, Mwangi B, Amaral-Silva H, Tannous J, Wu MJ, et al. Hippocampal subfield volumes in mood disorders. Mol Psychiatry. 2017; 22(9):1352-8. https://doi.org/10.1038/mp.2016.262.

11. Cao XH, Liu ZF, Xu C, Li JY, Gao Q, Sun N, et al. Disrupted resting-state functional connectivity of the hippocampus in medication-naive patients with major depressive disorder. J Affect Disord. 2012;141(2-3):194-203. https://doi.org/10.1016/j.jad.2012.03.002.

12. Carter RM, O'Doherty JP, Seymour B, Koch C, Dolan RJ. Contingency awareness in human aversive conditioning involves the middle frontal gyrus. Neuroimage. 2006;29(3):1007-12. https://doi.org/10.1016/j. neuroimage.2005.09.011.

13. Chabardes S, Kahane P, Minotti L, Hoffmann D, Benabid AL. Anatomy of the temporal pole region. Epileptic Disord. 2002;4:S9-S15.

14. de la Vega A, Chang LJ, Banich MT, Wager TD, Yarkoni T. Large-scale metaanalysis of human medial frontal cortex reveals tripartite functional organization. J Neurosci. 2016;36(24):6553-62. https://doi.org/10.1523/ JNEUROSCI.4402-15.2016.

15. Drabant EM, Kuo JR, Ramel W, Blechert J, Edge MD, Cooper JR, et al. Experiential, autonomic, and neural responses during threat anticipation vary as a function of threat intensity and neuroticism. Neuroimage. 2011; 55(1):401-10. https://doi.org/10.1016/j.neuroimage.2010.11.040.

16. Du MY, Wu QZ, Yue Q, Li J, Liao Y, Kuang WH, et al. Voxelwise meta-analysis of gray matter reduction in major depressive disorder. Prog NeuroPsychopharmacol Biol Psychiatry. 2012;36(1):11-6. https://doi.org/10.1016/j. pnpbp.2011.09.014

17. Duvernoy HM, Cattin E, Naidich T, Fatterpekar GM, Raybaud C, Risold PY, Sakvolini U, Scarabino T. The human Hippocampus. Berlin Heidelberg: Springer Verlag; 2005.

18. Eichenbaum H. Hippocampus: remembering the choices. Neuron. 2013; 77(6):999-1001. https://doi.org/10.1016/j.neuron.2013.02.034.

19. Fateh AA, Long ZL, Duan XJ, Cui Q, Pang YJ, Farooq MU, et al. Hippocampal functional connectivity-based discrimination between bipolar and major depressive disorders. Psychiatry Res Neuroimaging. 2019;284:53-60. https:// doi.org/10.1016/j.pscychresns.2019.01.004
20. Gallucci A, Riva P, Romero Lauro LJ, Bushman BJ. Stimulating the ventrolateral prefrontal cortex (VLPFC) modulates frustration-induced aggression: a tDCS experiment. Brain Stimul. 2019. https://doi.org/10.1016/j. brs.2019.10.015.

21. Geng H, Wu F, Kong L, Tang Y, Zhou Q, Chang M, et al. Disrupted structural and functional connectivity in prefrontal-Hippocampus circuitry in firstepisode medication-naive adolescent depression. PLoS One. 2016;11(2): e0148345. https://doi.org/10.1371/journal.pone.0148345.

22. Grafton ST. The cognitive neuroscience of prehension: recent developments. Exp Brain Res. 2010;204(4):475-91. https://doi.org/10.1007/ s00221-010-2315-2.

23. Guo WB, Liu F, Xiao CQ, Zhang ZK, Liu JR, Yu MY, et al. Decreased insular connectivity in drug-naive major depressive disorder at rest. J Affect Disord. 2015;179:31-7. https://doi.org/10.1016/j.jad.2015.03.028.

24. Hamilton, M. A rating scale for depression. J Neurol Neurosurg Psychiatry. 1960;23:56-62. https://doi.org/10.1136/jnnp.23.1.56

25. Han KM, Won E, Sim Y, Tae WS. Hippocampal subfield analysis in medication-naive female patients with major depressive disorder. J Affect Disord. 2016;194:21-9. https://doi.org/10.1016/j.jad.2016.01.019.

26. Huang Y, Coupland NJ, Lebel RM, Carter R, Seres P, Wilman AH, Malykhin NV. Structural changes in hippocampal subfields in major depressive disorder: a high-field magnetic resonance imaging study. Biol Psychiatry. 2013;74(1):62-8. https://doi.org/10.1016/j.biopsych.2013.01.005.

27. Japee S, Holiday K, Satyshur MD, Mukai I, Ungerleider LG. A role of right middle frontal gyrus in reorienting of attention: a case study. Front Syst Neurosci. 2015:9:23. https://doi.org/10.3389/fnsys.2015.00023.

28. Jeltsch H, Bertrand F, Lazarus C, Cassel JC. Cognitive performances and locomotor activity following dentate granule cell damage in rats: role of lesion extent and type of memory tested. Neurobiol Learn Mem. 2001;76(1): 81-105. https://doi.org/10.1006/nlme.2000.3986.

29. Johnson A, Redish AD. Neural ensembles in CA3 transiently encode paths forward of the animal at a decision point. J Neurosci. 2007:27(45):12176-89. https://doi.org/10.1523/Jneurosci.3761-07.2007.

30. Kaiser RH, Andrews-Hanna JR, Wager TD, Pizzagalli DA. Large-scale network dysfunction in major depressive disorder: a meta-analysis of resting-state functional connectivity. JAMA Psychiatry. 2015;72(6):603-11. https://doi.org/ 10.1001/jamapsychiatry.2015.0071.

31. Karl JM, Whishaw IQ. Different evolutionary origins for the reach and the grasp: an explanation for dual visuomotor channels in primate parietofrontal cortex. Front Neurol. 2013;4:208. https://doi.org/10.3389/fneur.2013.00208.

32. Kelley R, Garrett A, Cohen J, Gomez R, Lembke A, Keller J, et al. Altered brain function underlying verbal memory encoding and retrieval in psychotic major depression. Psychiatry Res. 2013;211(2):119-26. https://doi.org/10. 1016/j.pscychresns.2012.06.008.

33. Kesner RP, Lee I, Gilbert P. A behavioral assessment of hippocampal function based on a subregional analysis. Rev Neurosci. 2004;15(5):333-51.

34. Kessler RC. The costs of depression. Psychiatr Clin North Am. 2012;35(1):1 14. https://doi.org/10.1016/j.psc.2011.11.005

35. Kishi T, Tsumori T, Yokota S, Yasui Y. Topographical projection from the hippocampal formation to the amygdala: a combined anterograde and retrograde tracing study in the rat. J Comp Neurol. 2006;496(3):349-68. https://doi.org/10.1002/cne.20919.

36. Kitada R, Johnsrude IS, Kochiyama T, Lederman SJ. Brain networks involved in haptic and visual identification of facial expressions of emotion: an fMRI study. Neuroimage. 2010;49(2):1677-89. https://doi.org/10.1016/j. neuroimage.2009.09.014

37. Leaver AM, Espinoza R, Joshi SH, Vasavada M, Njau S, Woods RP, Narr KL. Desynchronization and plasticity of Striato-frontal connectivity in major depressive disorder. Cereb Cortex. 2016;26(11):4337-46. https://doi.org/10. 1093/cercor/bhv207.

38. Leech $\mathrm{R}$, Sharp DJ. The role of the posterior cingulate cortex in cognition and disease. Brain. 2014;137:12-32. https://doi.org/10.1093/brain/awt162.

39. Liang PP, Wang ZQ, Yang YH, Jia XQ, Li KC. Functional disconnection and compensation in mild cognitive impairment: evidence from DLPFC connectivity using resting-state fMRI. PLoS One, 6(7). Doi: ARTN e22153. 2011. https://doi.org/10.1371/journal.pone.0022153.

40. Malykhin NV, Coupland NJ. Hippocampal neuroplasticity in major depressive disorder. Neuroscience. 2015:309:200-13. https://doi.org/10.1016/j. neuroscience.2015.04.047.

41. Mayberg HS. Limbic-cortical dysregulation: a proposed model of depression. J Neuropsychiatry Clin Neurosci. 1997;9(3):471-81. https://doi.org/10.1176/jnp.9.3.471. 
42. Mazurek KA, Schieber MH. Injecting instructions into premotor cortex Neuron. 2017;96(6):1282. https://doi.org/10.1016/j.neuron.2017.11.006.

43. Merikangas KR, Ames M, Cui L, Stang PE, Ustun TB, Von Korff M, Kessler RC. The impact of comorbidity of mental and physical conditions on role disability in the US adult household population. Arch Gen Psychiatry. 2007; 64(10):1180-8. https://doi.org/10.1001/archpsyc.64.10.1180.

44. Milne AM, MacQueen GM, Hall GB. Abnormal hippocampal activation in patients with extensive history of major depression: an fMRI study. J Psychiatry Neurosci. 2012;37(1):28-36. https://doi.org/10.1503/jpn.110004.

45. Mulders PC, van Eijndhoven PF, Schene AH, Beckmann CF, Tendolkar I. Resting-state functional connectivity in major depressive disorder: a review. Neurosci Biobehav Rev. 2015a;56:330-44. https://doi.org/10.1016/j.neubiorev. 2015.07.014

46. Ng M, Fleming T, Robinson M, Thomson B, Graetz N, Margono C, et al. Global, regional, and national prevalence of overweight and obesity in children and adults during 1980-2013: a systematic analysis for the global burden of disease study 2013. Lancet. 2014;384(9945):766-81. https://doi. org/10.1016/s0140-6736(14)60460-8.

47. O'Mara SM, Commins $S$, Anderson M, Gigg J. The subiculum: a review of form, physiology and function. Prog Neurobiol. 2001;64(2):129-55.

48. Ohira H, Nomura M, Ichikawa $N$, Isowa $T$, lidaka $T$, Sato A, et al. Association of neural and physiological responses during voluntary emotion suppression. Neuroimage. 2006;29(3):721-33. https://doi.org/10.1016/j. neuroimage.2005.08.047.

49. Ongur $\mathrm{D}$, Price $\mathrm{J}$. The organization of networks within the orbital and medial prefrontal cortex of rats, monkeys and humans. Cerebral Cortex, 10(3), 206-219. Doi. 2000. https://doi.org/10.1093/cercor/10.3.206.

50. Owen AM, McMillan KM, Laird AR, Bullmore E. N-back working memory paradigm: a meta-analysis of normative functional neuroimaging studies. Hum Brain Mapp. 2005;25(1):46-59. https://doi.org/10.1002/hbm.20131.

51. Phillips ML, Drevets WC, Rauch SL, Lane R. Neurobiology of emotion perception II: implications for major psychiatric disorders. Biol Psychiatry. 2003;54(5):515-28.

52. Pitkanen A, Pikkarainen M, Nurminen N, Ylinen A. Reciprocal connections between the amygdala and the hippocampal formation, perirhinal cortex, and postrhinal cortex in rat - a review. Parahippocampal Region. 2000;911:369-91.

53. Qin SZ, Duan XJ, Supekar K, Chen HF, Chen TW, Menon V. Large-scale intrinsic functional network organization along the long axis of the human medial temporal lobe. Brain Struct Funct. 2016;221(6):3237-58. https://doi. org/10.1007/s00429-015-1098-4

54. Ridderinkhof KR, van den Wildenberg WP, Segalowitz SJ, Carter CS. Neurocognitive mechanisms of cognitive control: the role of prefrontal cortex in action selection, response inhibition, performance monitoring, and reward-based learning. Brain Cogn. 2004;56(2):129-40. https://doi.org/10. 1016/j.bandc.2004.09.016.

55. Rudebeck PH, Murray EA. The orbitofrontal Oracle: cortical mechanisms for the prediction and evaluation of specific behavioral outcomes. Neuron. 2014;84(6):1143-56. https://doi.org/10.1016/j.neuron.2014.10.049.

56. Sampath D, Sathyanesan M, Newton SS. Cognitive dysfunction in major depression and Alzheimer's disease is associated with hippocampalprefrontal cortex dysconnectivity. Neuropsychiatr Dis Treat. 2017;13:1509-19. https://doi.org/10.2147/NDT.S136122.

57. Shahnazian D, Holroyd CB. Distributed representations of action sequences in anterior cingulate cortex: a recurrent neural network approach. Psychon Bull Rev. 2018;25(1):302-21. https://doi.org/10.3758/s13423-017-1280-1.

58. Sheline YI. Depression and the hippocampus: cause or effect? Biol Psychiatry. 2011;70(4):308-9. https://doi.org/10.1016/j.biopsych.2011.06.006.

59. Sheline YI, Liston C, McEwen BS. Parsing the Hippocampus in depression: chronic stress, hippocampal volume, and major depressive disorder. Biol Psychiatry. 2019;85(6):436-8. https://doi.org/10.1016/j.biopsych.2019.01.011.

60. Simmons WK, Avery JA, Barcalow JC, Bodurka J, Drevets WC, Bellgowan P. Keeping the body in mind: insula functional organization and functional connectivity integrate interoceptive, Exteroceptive, and emotional awareness. Hum Brain Mapp. 2013;34(11):2944-58. https://doi.org/10.1002/hbm.22113.

61. Song XW, Dong ZY, Long XY, Li SF, Zuo XN, Zhu CZ, Zang YF. REST: a toolkit for resting-state functional magnetic resonance imaging data processing. PloS one. 2011;6(9):e25031.

62. Takamura T, Hanakawa T. Clinical utility of resting-state functional connectivity magnetic resonance imaging for mood and cognitive disorders. J Neural Transm (Vienna). 2017;124(7):821-39. https://doi.org/10. 1007/s00702-017-1710-2.
63. Tao H, Guo S, Ge T, Kendrick KM, Xue Z, Liu Z, Feng J. Depression uncouples brain hate circuit. Mol Psychiatry. 2013;18(1):101-11. https://doi. org/10.1038/mp.2011.127.

64. Tian Y, Zalesky A, Bousman C, Everall I, Pantelis C. Insula functional connectivity in schizophrenia: subregions, gradients, and symptoms. Biol Psychiatry Cogn Neurosci Neuroimaging. 2019;4(4):399-408. https://doi.org/ 10.1016/j.bpsc.2018.12.003.

65. Trimper JB, Galloway CR, Jones AC, Mandi K, Manns JR. Gamma oscillations in rat hippocampal subregions dentate Gyrus, CA3, CA1, and Subiculum underlie associative memory encoding. Cell Rep. 2017;21(9):2419-32. https:// doi.org/10.1016/j.celrep.2017.10.123.

66. Veer IM, Oei NYL, Spinhoven P, van Buchem MA, Elzinga BM, Rombouts SARB. Beyond acute social stress: increased functional connectivity between amygdala and cortical midline structures. Neuroimage. 2011;57(4):1534-41. https://doi.org/10.1016/j.neuroimage.2011.05.074.

67. Veltman DJ, Rombouts SA, Dolan RJ. Maintenance versus manipulation in verbal working memory revisited: an fMRI study. Neuroimage. 2003:18(2):247-56.

68. Wang C, Wu HW, Chen FF, Xu JP, Li HM, Li H, Wang JJ. Disrupted functional connectivity patterns of the insula subregions in drug-free major depressive disorder. J Affect Disord. 2018;234:297-304. https://doi.org/10.1016/j.jad. 2017.12.033.

69. Wang L, Hermens DF, Hickie IB, Lagopoulos J. A systematic review of resting-state functional-MRI studies in major depression. J Affect Disord. 2012:142(1-3):6-12. https://doi.org/10.1016/j.jad.2012.04.013.

70. Wang Z, Yuan Y, Bai F, Shu H, You J, Li L, Zhang Z. Altered functional connectivity networks of hippocampal subregions in remitted late-onset depression: a longitudinal resting-state study. Neurosci Bull. 2015;31(1):1321. https://doi.org/10.1007/s12264-014-1489-1.

71. Wikenheiser AM, Schoenbaum G. Over the river, through the woods: cognitive maps in the hippocampus and orbitofrontal cortex. Nat Rev Neurosci. 2016;17(8):513-23. https://doi.org/10.1038/nrn.2016.56.

72. Wisse LEM, Biessels GJ, Stegenga BT, Kooistra M, van der Veen PH, Zwanenburg JJM, et al. Major depressive episodes over the course of 7 years and hippocampal subfield volumes at 7 tesla MRI: the PREDICT-MR study. J Affect Disord. 2015;175:1-7. https://doi.org/10.1016/j.jad.2014.12.052.

73. World Health Organization. Depression and other common mental disorders: global health estimates. 2017.

74. Zeidman P, Lutti A, Maguire EA. Investigating the functions of subregions within anterior hippocampus. Cortex. 2015;73:240-56. https://doi.org/10. 1016/j.cortex.2015.09.002

75. Zeng LL, Shen H, Liu L, Hu DW. Unsupervised classification of major depression using functional connectivity MRI. Hum Brain Mapp. 2014;35(4): 1630-41. https://doi.org/10.1002/hbm.22278.

76. Zhang $\mathrm{H}$, Ji J. Prevalence of mental disorders in China. Lancet Psychiatry. 2019;6(3):188-9. https://doi.org/10.1016/s2215-0366(19)30034-3.

77. Zhang HW, Li L, Wu M, Chen ZQ, Hu XY, Chen Y, et al. Brain gray matter alterations in first episodes of depression: a meta-analysis of whole-brain studies. Neurosci Biobehav Rev. 2016;60:43-50. https://doi.org/10.1016/j. neubiorev.2015.10.011

78. Zhong Y, Zhang R, Li K, et al. Altered cortical and subcortical local coherence in PTSD: evidence from resting-state fMRI. Acta Radiol. 2015; 56(6):746-53.

79. Zuo, Z. W., Ran, S. H., Wang, Y., Li, C., Han, Q., Tang, Q. Y., ... Li, H. T. (2018). Altered structural covariance among the dorsolateral prefrontal cortex and amygdala in treatment-naive patients with major depressive disorder. Front Psychiatry, 9. Doi: ARTN 32310.3389/fpsyt.2018.00323.

\section{Publisher's Note}

Springer Nature remains neutral with regard to jurisdictional claims in published maps and institutional affiliations. 